OPEN ACCESS

Edited by:

Salvatore Salomone,

University of Catania, Italy

Reviewed by:

Frank Kloprogge,

University College London,

United Kingdom

Jotam G. Pasipanodya,

Texas Tech University, United States

*Correspondence:

Denise E. Kirschner kirschne@umich.edu

Jennifer J. Linderman

linderma@umich.edu

Specialty section:

This article was submitted to Experimental Pharmacology and Drug Discovery,

a section of the journal

Frontiers in Pharmacology

Received: 27 November 2019 Accepted: 06 March 2020

Published: 24 March 2020

Citation:

Cicchese JM, Dartois V, Kirschner DE and Linderman JJ (2020) Both

Pharmacokinetic Variability and

Granuloma Heterogeneity Impact the Ability of the First-Line Antibiotics to Sterilize Tuberculosis Granulomas.

Front. Pharmacol. 11:333. doi: 10.3389/fphar.2020.00333

\section{Both Pharmacokinetic Variability and Granuloma Heterogeneity Impact the Ability of the First-Line Antibiotics to Sterilize Tuberculosis Granulomas}

\author{
Joseph M. Cicchese ${ }^{1}$, Véronique Dartois ${ }^{2,3}$, Denise E. Kirschner ${ }^{4 *}$ \\ and Jennifer J. Linderman ${ }^{1 *}$ \\ ${ }^{1}$ Department of Chemical Engineering, University of Michigan, Ann Arbor, MI, United States, 2 Public Health Research \\ Institute, New Jersey Medical School, Rutgers, The State University of New Jersey, Newark, NJ, United States, ${ }^{3}$ Center for \\ Discovery and Innovation, Hackensack Meridian Health, Nutley, NJ, United States, ${ }^{4}$ Department of Microbiology and \\ Immunology, University of Michigan Medical School, Ann Arbor, MI, United States
}

Tuberculosis (TB) remains as one of the world's deadliest infectious diseases despite the use of standardized antibiotic therapies. Recommended therapy for drug-susceptible TB is up to 6 months of antibiotics. Factors that contribute to lengthy regimens include antibiotic underexposure in lesions due to poor pharmacokinetics (PK) and complex granuloma compositions, but it is difficult to quantify how individual antibiotics are affected by these factors and to what extent these impact treatments. We use our next-generation multi-scale computational model to simulate granuloma formation and function together with antibiotic pharmacokinetics and pharmacodynamics, allowing us to predict conditions leading to granuloma sterilization. In this work, we focus on how PK variability, determined from human PK data, and granuloma heterogeneity each quantitatively impact granuloma sterilization. We focus on treatment with the standard regimen for TB of four first-line antibiotics: isoniazid, rifampin, ethambutol, and pyrazinamide. We find that low levels of antibiotic concentration due to naturally occurring PK variability and complex granulomas leads to longer granuloma sterilization times. Additionally, the ability of antibiotics to distribute in granulomas and kill different subpopulations of bacteria contributes to their specialization in the more efficacious combination therapy. These results can inform strategies to improve antibiotic therapy for TB.

Keywords: agent-based model, pharmacokinetic/pharmacodynamic (PK/PD) model, multi-scale model, isoniazid, rifampin, tissue distribution

\section{INTRODUCTION}

Tuberculosis (TB) continues to be one of the world's deadliest infectious diseases, leading to the death of 1.3 million people in 2017, about 2-3 people per minute (Global tuberculosis report, 2018). Caused by infection with the pathogen Mycobacterium tuberculosis (Mtb), TB most commonly presents as a pulmonary disease in adults when individuals inhale aerosolized Mtb transmitted by 
other infected individuals. The immune response in the lungs leads to the formation of multiple lesions called granulomas, collections of immune cells that act to contain the infection both immunologically and physically but also present a barrier to antibiotic diffusion and delivery (Ramakrishnan, 2012; Dartois, 2014; Pienaar et al., 2015a). Understanding penetration and distribution of antibiotics in granulomas is critical to understanding how best to treat TB.

The current recommended regimen to treat active, drugsusceptible $\mathrm{TB}$ disease requires up to six months of multiple antibiotics (Nahid et al., 2016). For the first two months, patients take daily doses of isoniazid (INH, H), rifampin (RIF, R), ethambutol (EMB, E), and pyrazinamide (PZA, Z), referred to as HRZE. Each of these antibiotics has side effects associated with their use that, together with the lengthy treatment duration, make it difficult for patients to properly adhere to the regimen (Yee et al., 2003; Munro et al., 2007). Efforts such as directly observed therapy (DOT) attempt to increase patient adherence but are not tractable on a global scale (Steffen et al., 2010; McLaren et al., 2016). Emergence of multidrug-resistant (defined as resistant to INH and RIF) and extensively drugresistant TB (resistant to INH, RIF, and a second-line injectable) further complicates treatment (Global tuberculosis report, 2018). There is a need for improved antibiotic therapy for TB and to understand what causes treatment failure.

There are two key factors outside of drug resistance that have been identified as contributing to drug failure in TB: pharmacokinetic (PK) variability (acting at population scale) and granuloma heterogeneity (acting at the host scale). How these factors interact to affect both the rate and extent of sterilization during treatment is not well-understood. PK variability is defined as differences in plasma antibiotic exposure, typically measured as variability in plasma area under the curve (AUC) measurements. Population PK models can help determine appropriate dosing of TB antibiotics and represent this variability based on distributions of PK model parameters (Zhu et al., 2004; Jonsson et al., 2011; Denti et al., 2015; Lalande et al., 2015). These distributions can be related to natural differences in populations through covariates such as weight, age, or overall health. Additionally, PK variability can be due to differences at the genetic scale, such as in $\mathrm{N}$ acetyltransferase 2 involved in the metabolism of INH (Blum et al., 1991; Kinzig-Schippers et al., 2005). This PK variability can lead to poor exposure in granuloma lesions, reducing the amount of time antibiotic concentrations are above therapeutic thresholds during therapy (Strydom et al., 2019).

Host-scale heterogeneity encompasses host-level variations in granuloma number, size, and composition. Granuloma size and composition can lead to slower diffusion of antibiotics, spatial gradients of concentration, and underexposure at the host tissue scale (Dartois, 2014; Pienaar et al., 2015a; Prideaux et al., 2015; Sarathy et al., 2016). Granuloma composition can affect how antibiotics accumulate or fail to accumulate within lesions (Dartois, 2014). For example, EMB's clinical efficacy may partially be explained by its ability to accumulate in cellular regions of the granuloma (Zimmerman et al., 2017). Structural differences in lesions affect the sterilizing ability of PZA, as shown in different strains of mice (Irwin et al., 2016). Caseous regions of the granuloma may also harbor bacteria that are phenotypically more tolerant, and may be less accessible, to many TB antibiotics (Sarathy et al., 2018).

Capturing both PK variability at the population scale and granuloma heterogeneity at the host scale in a computational model can help predict granuloma sterilization and design antibiotic regimens. Our group previously developed a computational model that incorporates the host formation of granulomas and antibiotic PK to predict the sterilization of granulomas using different regimens with INH and RIF (Pienaar et al., 2015a; Pienaar et al., 2015b). Using this multi-scale, systems pharmacology model, we have also highlighted major differences between members of the fluoroquinolone drug class and simulated TB therapy with development of antibiotic resistance (Pienaar et al., 2017; Pienaar et al., 2018). Using this computational framework provides a way to include both $\mathrm{PK}$ variability and granuloma heterogeneity to predict whether a treatment can achieve granuloma sterilization in primary, pulmonary TB in adults.

Here we use our hybrid, multi-scale agent-based model to capture PK variability and granuloma heterogeneity and to simulate antibiotic treatment of primary, lung granulomas. For the first time with this model, we simulate treatment based on human PK and with the combination of the four first-line antibiotics used to treat TB: INH, RIF, EMB, and PZA. We also present a sequential calibration scheme that captures spatial distributions of antibiotics within granulomas and known PK variability that exists across the population scale and at the host scale within granulomas. Using this highly detailed model, we discuss the role of first-line antibiotics (HRZE) in sterilizing granulomas and how PK variability and granuloma heterogeneity impact distributions of sterilization times.

\section{METHODS}

\section{Computational Model of Granuloma Formation and Function}

GranSim is a well-established hybrid, multi-scale computational model that produces the emergent behavior of granuloma formation in Mtb infection (Segovia-Juarez et al., 2004; FallahiSichani et al., 2011; Cilfone et al., 2013; Linderman et al., 2015). Briefly, this agent-based model simulates immune cell movement and interactions, and bacterial growth on a spatial grid representing an area of lung tissue. The immune cell agents, such as different classes of macrophages and T cells, move in response to chemokine gradients and interact with each other according to immunology-derived rules to activate or deactivate immune cells/responses. Bacteria in the model are simulated as individual agents, and they exist in three distinct subpopulations: extracellular replicating, extracellular non-replicating, or intracellular (inside macrophages). The effective growth rate of bacteria in these three subpopulations is influenced by extracellular or intracellular location and availability of nutrients and oxygen (Pienaar et al., 2016). Non-replicating Mtb represent bacteria trapped within caseum, which presents 
hypoxic conditions with limited nutrient resources (Nathan and Barry III, 2015; Sarathy et al., 2018). A more detailed explanation of GranSim and the simulation rules and assumptions can be found online (http://malthus.micro.med.umich.edu/GranSim/). GranSim simulates lung granulomas that form due to primary, pulmonary infection in adults and captures a wide diversity of granulomas through variations in host immune system parameters and stochastic events in the agent-based model. The boundary of the granuloma is defined by regions of high cell density, and outlines of the granuloma are drawn to enclose these regions. Parameters that were varied to generate our library of heterogeneous granulomas are listed in Table $\mathbf{1 .}$

TABLE 1 | Host immune parameters for in silico granulomas. Timestep units represent 10-min time steps in the agent-based simulation. Parameter values based on previously published work (Pienaar et al., 2015a).

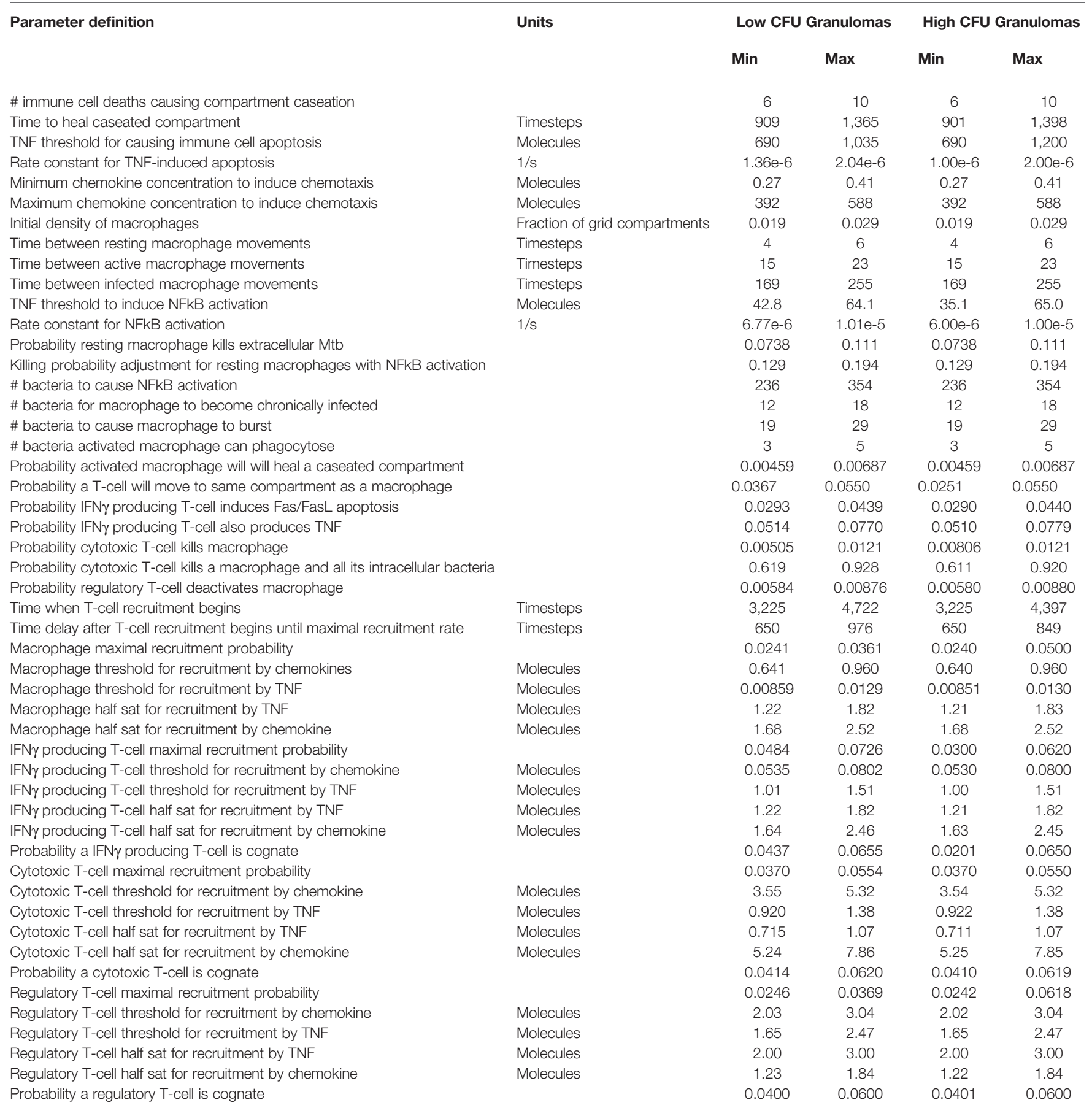




\section{In Silico Granuloma Library}

We generate two distinct libraries of granulomas that are heterogeneous in bacterial load and cellular composition: one categorized as low-CFU (colony-forming unit, equal to the number of bacteria in the simulation) granulomas and the other as high-CFU granulomas. The low-CFU granulomas are smaller in size and have CFU/granuloma that are more stable over time, whereas the high-CFU granulomas are larger in size, have increasing CFU over time, and have higher levels of caseum. To generate the low-CFU granulomas, we sampled 500 parameter sets based on ranges for host immune system parameters listed in Table 1 using Latin Hypercube Sampling (LHS) (Pienaar et al., 2015a). Using the simulation outputs at day 300 for granuloma size and CFU, we performed sensitivity analysis using partial rank correlation coefficients to determine parameters that have the most significant impact on those two outcomes (Marino et al., 2008). A total of 400 high-CFU granulomas were generated by increasing or decreasing the upper and lower bounds of the parameter ranges that have the strongest correlation with granuloma size and CFU, as well as initializing simulations with multiple infection locations to generate larger granulomas. Parameter ranges for all granulomas are shown in Table 1. Low-CFU granulomas are simulated on a 200 by 200 compartment square grid representing a 4 by $4 \mathrm{~mm}$ section of lung tissue (each grid compartment has a side-length of 20 microns), whereas the high-CFU are run on 300 by 300 compartment grid representing 6 by $6 \mathrm{~mm}$. Note that we simulate the small granulomas on a smaller grid for computational efficiency, as the larger is not required. At day 300 , a total of 354 low-CFU granulomas and 352 high-CFU granulomas still had bacteria and were selected for treatment simulations. Figure 1 shows CFU per granuloma of these two groups.

\section{Plasma Pharmacokinetic Model}

The plasma PK model is comprised of a two-compartmental model with one or two transit compartments that simulate oral absorption. INH and RIF follow a two-absorption compartment model based on previously developed PK models, whereas EMB and PZA are simulated with one absorption compartment based on best fits and other PK models (Jonsson et al., 2011; Kjellsson et al., 2012; Denti et al., 2015; Pienaar et al., 2015a; Zimmerman et al., 2017). The two-compartment model simulates distribution between plasma and peripheral tissue, and antibiotics are eliminated with a first-order clearance rate constant (Figure 2). Pharmacokinetic variability can be introduced by varying the parameters of the plasma $\mathrm{PK}$ model based on reported variability in the parameters (Table 2 ).

\section{Tissue Pharmacokinetic Model}

The plasma PK model is linked to the agent-based environment through blood vessels placed on the simulation grid (Figure 2). Based on the difference between the plasma concentration and local tissue concentration in the compartments surrounding a blood vessel and the permeability of the antibiotic through blood vessel walls, a flux of antibiotic through the vessel wall is calculated as in previous work (Pienaar et al., 2015a). Antibiotics in the tissue (on the simulation grid) undergo a series of distribution events: diffusion, binding to extracellular material such as caseum, partitioning into macrophages, and

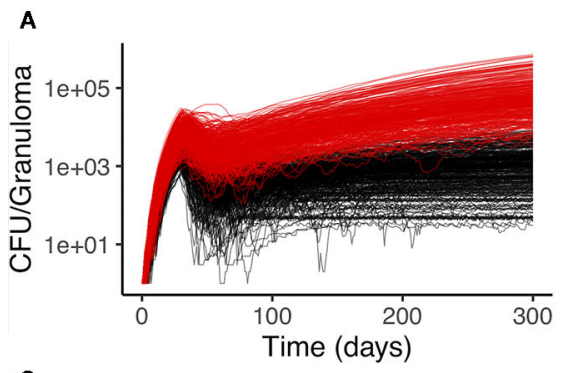

B
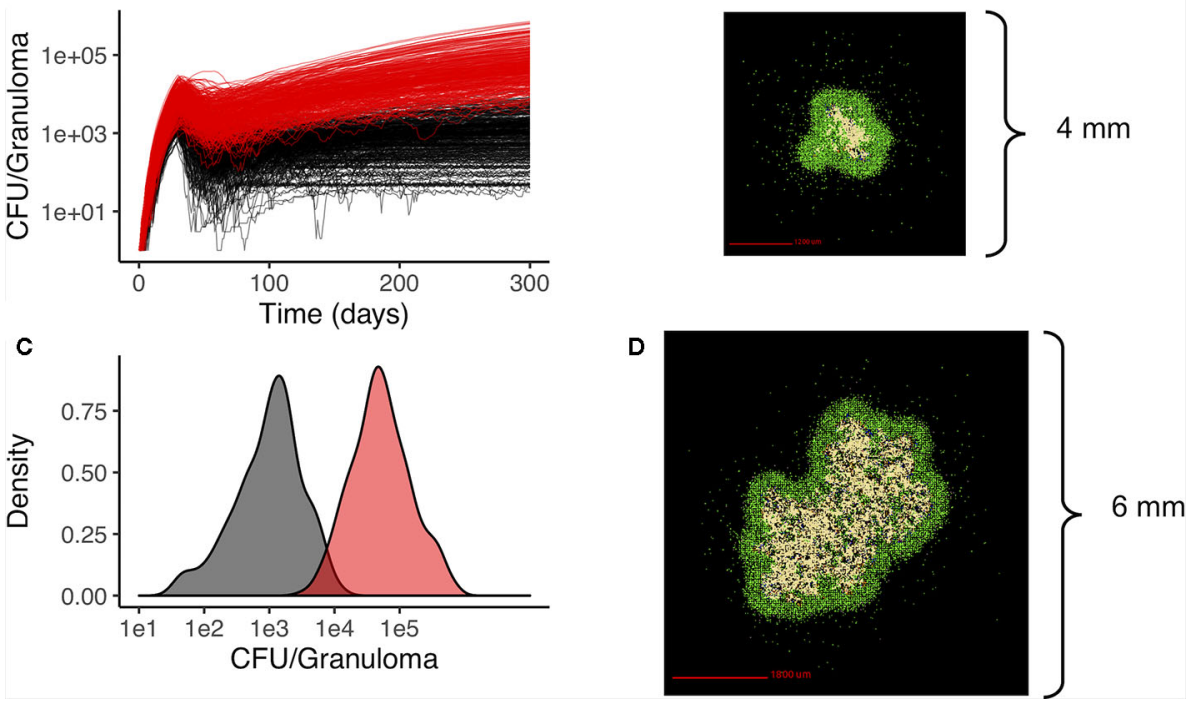

FIGURE 1 | Heterogeneous granulomas generated using the computational model GranSim. There are two groups of in silico granulomas at day 300 post infection: low CFU granulomas (black/gray, $n=354$ ) and high CFU granulomas (red, $n=352$ ). High CFU granulomas have increasing CFU over time relative to the more stable lower CFU granulomas (A). (C) shows the distribution of CFU per granuloma in the low CFU group (black) and the high CFU group (red) at day 300 . (B) shows an example of a low CFU in silico granuloma and (D) shows an example of a high CFU granuloma. In both simulations the colors represent: macrophages green; resting; blue, active; orange, infected; red, chronically infected), T cells (IFN-gamma producing; pink, cytotoxic, purple; regulatory, light blue), and caseated regions (tan). 


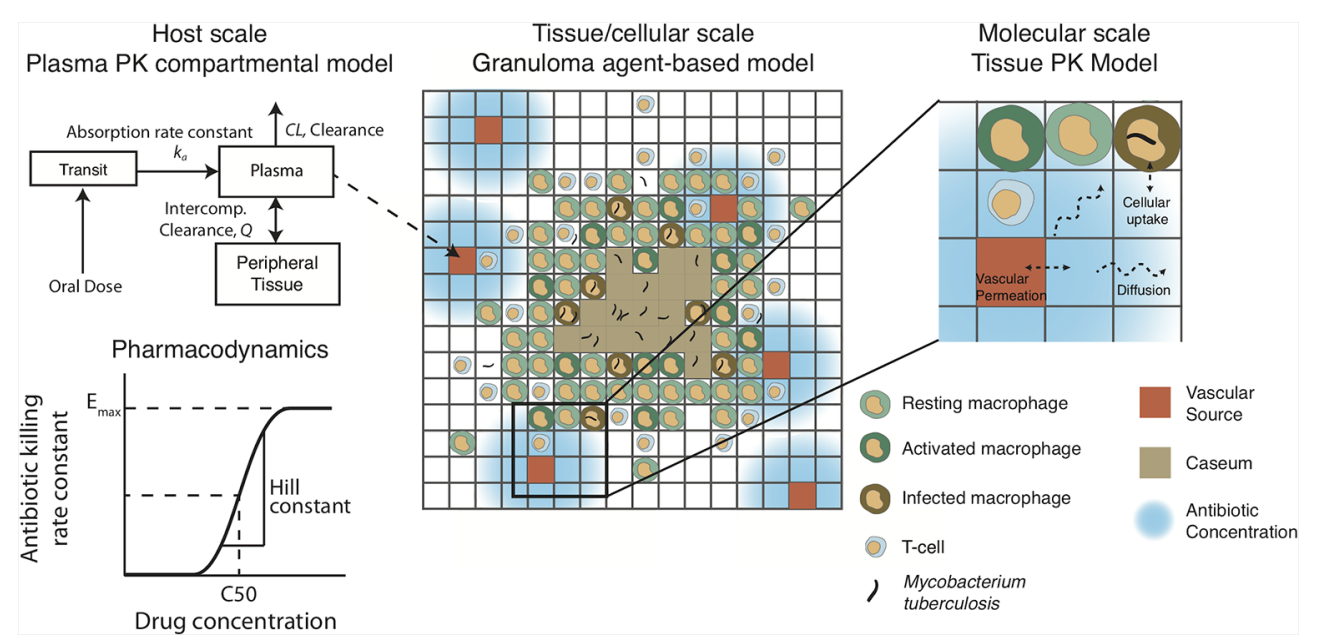

FIGURE 2 | Pharmacokinetic/pharmacodynamic dynamics in GranSim. Plasma concentration is simulated with a two-compartment pharmacokinetic (PK) model with one [ethambutol (EMB) and pyrazinamide (PZA)] or two [isoniazid (INH) and rifampin (RIF)] transit compartments to capture oral absorption. The amount of drug added or subtracted through the vascular sources in the agent-based spatial grid depends on local gradients of antibiotics. Antibiotics on the grid can diffuse, degrade, bind to extracellular material (such as caseum) and partition into macrophages. Based on intra- or extracellular concentrations in each grid compartment, a killing rate constant based on a Hill curve determines the probability per time step that a given bacterium will die due to exposure to antibiotics.

degradation. Implementation of vascular permeation, diffusion, binding and degradation is as previously published (Cilfone et al., 2015; Pienaar et al., 2015a). Antibiotics on the simulation grid can be tracked as free molecules, bound to extracellular material, or partitioned into macrophages. When calibrating and fitting to data, we use total drug concentration in a grid compartment, but only free or intracellular antibiotic is used to determine antimicrobial activity, depending on the location of bacteria. Calibrated tissue PK parameters are given in Table 3 (see below for calibration datasets).

\section{Sequential Pharmacokinetic Model Calibration Scheme}

Gradients between plasma and tissue concentrations drive the amount of antibiotic delivered into the agent-based model simulation through blood vessels, so fitting tissue PK parameters to match the experimentally observed spatial distribution and average antibiotic concentrations in granuloma lesions requires incorporating both plasma PK variability and granuloma heterogeneity. We have devised a pipeline for incorporating these factors into GranSim (Figure

TABLE 2 | Plasma pharmacokinetic parameters listed with the ranges used to calibrate the tissue pharmacokinetic parameters, as well as the parameter values for the average and low pharmacokinetic (PK) exposure treatment groups.

\begin{tabular}{|c|c|c|c|c|c|c|}
\hline Parameter & Units & Min & Max & Average PK & $\begin{array}{c}\text { Low } \\
\text { PK }\end{array}$ & Source \\
\hline INH Absorption rate constant & $1 / \mathrm{h}$ & 0.50 & 6.0 & 3.25 & 0.57 & Fit to data from (Prideaux et al., 2015) \\
\hline INH Intercompartmental clearance rate constant & L/(h*kg) & 0.20 & 0.70 & 0.45 & 0.67 & Fit to data from (Prideaux et al., 2015) \\
\hline INH Central compartment volume of distribution & $\mathrm{L} / \mathrm{kg}$ & 0.50 & 3.0 & 1.75 & 2.5 & Fit to data from (Prideaux et al., 2015) \\
\hline INH Peripheral compartment volume of distribution & L/kg & 25 & 40 & 32.5 & 37 & Fit to data from (Prideaux et al., 2015) \\
\hline INH Plasma clearance rate constant & $\mathrm{L} /\left(\mathrm{h}^{*} \mathrm{~kg}\right)$ & 0.0080 & 0.070 & 0.039 & 0.061 & Fit to data from (Prideaux et al., 2015) \\
\hline RIF Absorption rate constant & $1 / \mathrm{h}$ & 0.40 & 2.5 & 1.5 & 0.41 & Fit to data from (Prideaux et al., 2015) \\
\hline RIF Intercompartmental clearance rate constant & $\left\llcorner/\left(h^{*} k g\right)\right.$ & 2.0 & 5.9 & 3.9 & 3.95 & Fit to data from (Prideaux et al., 2015) \\
\hline RIF Central compartment volume of distribution & $\mathrm{L} / \mathrm{kg}$ & 0.18 & 0.57 & 0.38 & 0.48 & Fit to data from (Prideaux et al., 2015) \\
\hline RIF Peripheral compartment volume of distribution & $\mathrm{L} / \mathrm{kg}$ & 0.32 & 0.97 & 0.64 & 0.9 & Fit to data from (Prideaux et al., 2015) \\
\hline RIF Plasma clearance rate constant & $\mathrm{L} /\left(\mathrm{h}^{\star} \mathrm{kg}\right)$ & 0.050 & 0.30 & 0.175 & 0.3 & Fit to data from (Prideaux et al., 2015) \\
\hline EMB Absorption rate constant & $1 / \mathrm{h}$ & 0.10 & 0.80 & 0.45 & 0.1 & Fit to data from (Jonsson et al., 2011) \\
\hline EMB Intercompartmental clearance rate constant & $\mathrm{L} /\left(\mathrm{h}^{*} \mathrm{~kg}\right)$ & 0.45 & 0.70 & 0.57 & 0.56 & Fit to data from (Jonsson et al., 2011) \\
\hline EMB Central compartment volume of distribution & $\mathrm{L} / \mathrm{kg}$ & 0.80 & 1.95 & 1.37 & 1.7 & Fit to data from (Jonsson et al., 2011) \\
\hline EMB Peripheral compartment volume of distribution & L/kg & 8.1 & 12.7 & 10.4 & 12.6 & Fit to data from (Jonsson et al., 2011) \\
\hline EMB Plasma clearance rate constant & $\mathrm{L} /\left(\mathrm{h}^{*} \mathrm{~kg}\right)$ & 0.3 & 1.0 & 0.65 & 0.99 & Fit to data from (Jonsson et al., 2011) \\
\hline PZA Absorption rate constant & $1 / \mathrm{h}$ & 0.55 & 0.75 & 0.65 & 0.60 & Fit to data from (Prideaux et al., 2015) \\
\hline PZA Intercompartmental clearance rate constant & $\mathrm{L} /\left(\mathrm{h}^{\star} \mathrm{kg}\right)$ & 0.10 & 0.70 & 0.40 & 0.35 & Fit to data from (Prideaux et al., 2015) \\
\hline PZA Central compartment volume of distribution & L/kg & 0.25 & 0.75 & 0.50 & 0.74 & Fit to data from (Prideaux et al., 2015) \\
\hline PZA Peripheral compartment volume of distribution & $\mathrm{L} / \mathrm{kg}$ & 0.010 & 0.050 & 0.030 & 0.050 & Fit to data from (Prideaux et al., 2015) \\
\hline PZA Plasma clearance rate constant & $\mathrm{L} /\left(\mathrm{h}^{\star} \mathrm{kg}\right)$ & 0.010 & 0.050 & 0.030 & 0.050 & Fit to data from (Prideaux et al., 2015) \\
\hline
\end{tabular}


TABLE 3 | The calibrated tissue pharmacokinetic (PK) parameters for each antibiotic.

\begin{tabular}{|c|c|c|c|c|c|}
\hline Parameter & INH & RIF & EMB & PZA & Source \\
\hline Extracellular degradation rate constant (1/s) & $6.94 \mathrm{e}-8$ & $3.90 e-8$ & $1.73 e-8$ & $1.34 \mathrm{e}-8$ & Fit to data from (Prideaux et al., 2015; Zimmerman et al., 2017) \\
\hline Intraceullar degradation rate constant (1/s) & $2.84 \mathrm{e}-6$ & $2.59 \mathrm{e}-4$ & $8.75 e-6$ & $2.26 \mathrm{e}-3$ & Fit to data from (Prideaux et al., 2015; Zimmerman et al., 2017) \\
\hline Effective diffusivity ${ }^{*}\left(\mathrm{~cm}^{2} / \mathrm{s}\right)$ & $6.58 e-7$ & $5.08 \mathrm{e}-8$ & $5.20 \mathrm{e}-7$ & $3.24 \mathrm{e}-6$ & Fit to data from (Prideaux et al., 2015; Zimmerman et al., 2017) \\
\hline Cellular accumulation ratio & 1.13 & 24 & 5.95 & 0.593 & Fit to data from (Prideaux et al., 2015; Zimmerman et al., 2017) \\
\hline Vascular permeability (cm/s) & $1.34 \mathrm{e}-6$ & $2.65 \mathrm{e}-7$ & $1.33 e-7$ & $8.62 \mathrm{e}-6$ & Fit to data from (Prideaux et al., 2015; Zimmerman et al., 2017) \\
\hline Permeability coefficient & 0.25 & 3.3 & 7.4 & 1 & Fit to data from (Prideaux et al., 2015; Zimmerman et al., 2017) \\
\hline Fraction unbound to caseum & 1 & 0.052 & 0.35 & 1 & Fit to data from (Prideaux et al., 2015; Sarathy et al., 2016; Zimmerman et al., 2017) \\
\hline
\end{tabular}

*Guided by estimates from (Pruijn et al., 2008)

3A). Using our in silico granuloma library, each granuloma is assigned a different plasma PK set sampled using LHS from parameter ranges that capture biological variability (Table 2 ). Next, changes in antibiotic tissue concentrations over time are simulated for each granuloma with 200 tissue PK parameter sets sampled using LHS. For each tissue PK parameter set, the results from each granuloma are averaged at each time point, and then compared to experimental data. The tissue PK parameter set that both minimizes the sum of the squared error between average granuloma concentration and the experimental lesion

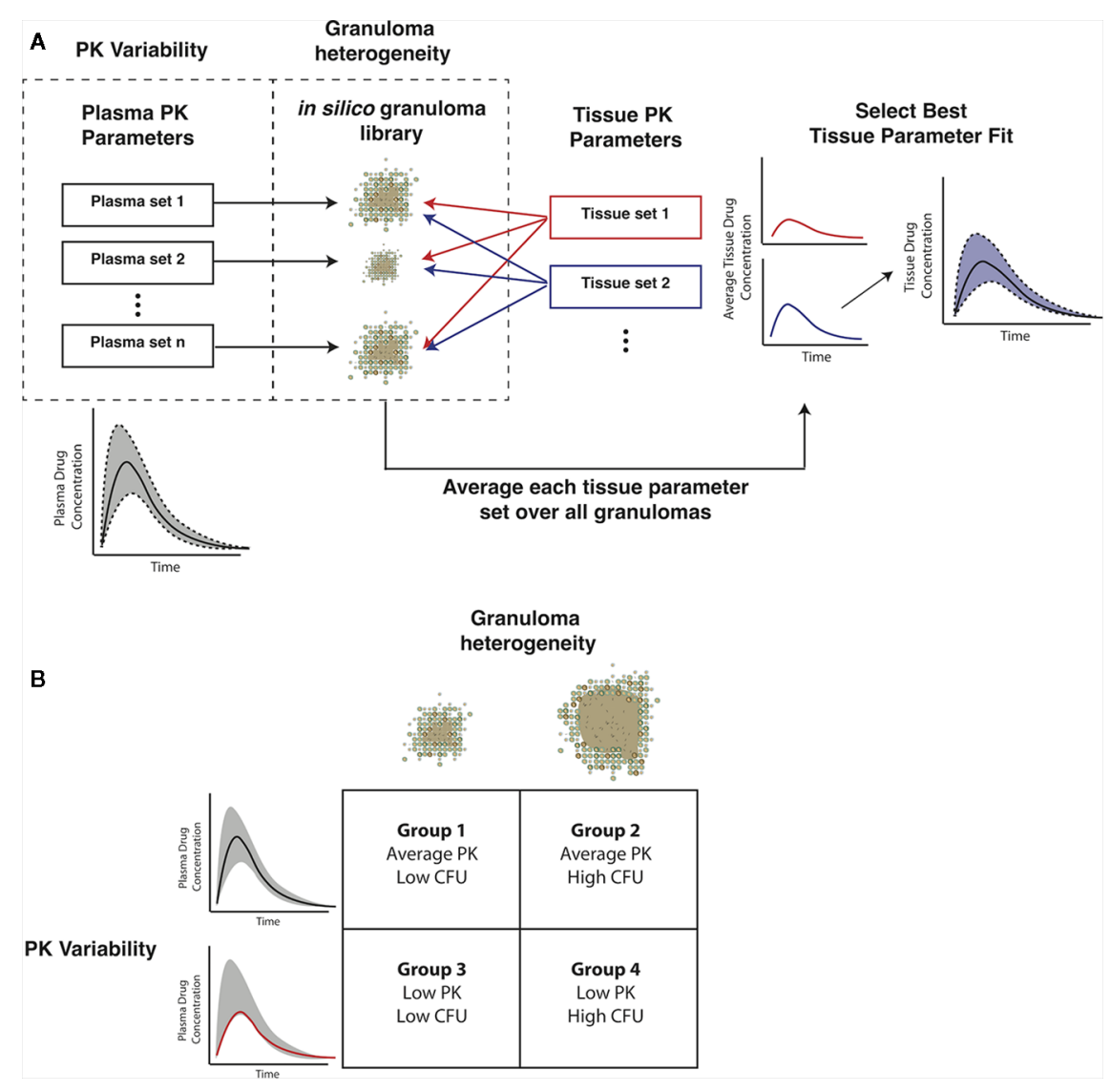

FIGURE 3 | Capturing pharmacokinetic variability and granuloma heterogeneity in pharmacokinetic (PK) calibration and treatment simulations. (A) shows our strategy. Based on population variability and ranges in plasma PK parameters, sets of plasma PK parameters are sampled and assigned to a set of in silico granulomas. Based on experimentally guided ranges for tissue PK parameters, a set of tissue PK parameters is obtained using Latin Hypercube Sampling (LHS). Simulations then predict antibiotic concentrations in the tissue. The average concentration over all granulomas for a given tissue PK parameter set is calculated and compared to experimental lesion concentrations. (B) shows the four types of treatment simulations that capture biologically relevant PK variability and granuloma heterogeneity: average PK exposure with low or high CFU granulomas and low PK exposure with low or high CFU granulomas. 
concentrations, as well as provides a good visual fit to the data is chosen as the calibrated tissue PK parameter set. Tissue PK parameter ranges for calibration sampling are listed in Table 3.

\section{Pharmacokinetic Data}

Plasma and tissue PK parameters for INH, RIF, and PZA are calibrated using plasma and lesion antibiotic concentrations measured in resected lung samples from patients with drugrefractory TB (Prideaux et al., 2015). EMB concentrations in rabbit TB granulomas are used to calibrate tissue PK parameters, based on rabbit plasma PK parameters (Zimmerman et al., 2017). Since tissue PK parameters are based on physical properties and interactions between drug molecules and tissue, we assume that tissue PK parameters in rabbits and humans are similar. To simulate human treatment with EMB, we replace the rabbit plasma PK parameters with human parameters fit to population PK measurements (Jonsson et al., 2011).

\section{Pharmacodynamic Model}

The pharmacodynamic model involves evaluating a concentration-dependent killing rate constant derived from a Hill curve:

$$
k=E_{\text {max }} \frac{C^{h}}{C^{h}+C_{50}^{h}}
$$

The killing rate constant $k$ (units of $1 /$ timestep or $1 / 10 \mathrm{~min}$ ) is dependent on the variable concentration $(C)$, the maximum killing rate constant $\left(E_{\max }\right)$, the concentration at half maximal killing $\left(C_{50}\right)$, and the Hill curve constant $(h)$. The concentration used to determine the antibiotic killing rate constant is based only on free drug concentration. Parameters $E_{\text {max }}, C_{50}$, and $h$ need to be determined for each antibiotic and for each bacterial subpopulation (replicating extracellular, non-replicating extracellular, and intracellular). To fit these parameters, we use in vitro dose-response assays from individual experiments from the literature of $\mathrm{Mtb}$ growth/death under varying antibiotic concentrations (see Table 4 for parameters and references for data and refer to Supplementary Figure 1 for calibrated fits to dose-response curves). In the present model, we do not include drug-drug interactions, so the highest single antibiotic killing rate constant for each antibiotic within a specific grid compartment in the simulation is used as the effective antibiotic killing rate constant for that location (Bhusal et al., 2005; Pienaar et al., 2015b). This assumption isolates the impact of PK variability and granuloma heterogeneity on granuloma sterilization within this study.

\section{In Silico Antibiotic Treatment of Granulomas}

Treatment simulations are executed by choosing the non-sterile set of low and high CFU in silico granulomas that have formed 300 days post infection (in the absence of antibiotics). The doses for the standard regimen are based on CDC recommended adult doses for each of the four antibiotics: INH, $5 \mathrm{mg} / \mathrm{kg}$; RIF, $10 \mathrm{mg} /$ $\mathrm{kg}$; EMB, $17 \mathrm{mg} / \mathrm{kg}$; PZA $21 \mathrm{mg} / \mathrm{kg}$ (Nahid et al., 2016). Simulated treatments use daily doses of each antibiotic. Treatment simulations are administered for a maximum of 180 days, which are based on standard regimen length (Nahid et al., 2016); simulations are stopped once granulomas sterilize to reduce computational resource use. After treatment, we calculate a simulated early bactericidal activity (EBA), which is defined as the rate of decrease of $\log _{10}$ (CFU) per day. For example, the EBA for $0-2$ days is calculated as $\left(\log _{10}\right.$ (CFU day $0)-\log _{10}(\mathrm{CFU}$ day 2$\left.)\right) / 2$. We simulate four groups of granulomas to incorporate $\mathrm{PK}$ variability and granuloma heterogeneity (Figure 3B). Group 1 has population average plasma PK exposure (AUC) with low-CFU granulomas and Group 2 has average PK exposure with high-CFU granulomas. Groups 3 and 4 both have low plasma PK exposure, with low and high-CFU granulomas respectively. Plasma PK parameter values for the low and average PK exposure are listed in Table 2.

\section{RESULTS}

\section{Pharmacokinetic Model Captures Plasma and Lesion Variability in Antibiotic Concentrations}

Heterogeneity in antibiotic exposure within granulomas is the result of two factors. Differences in plasma drug concentrations among individuals can be due to differences in drug absorption and elimination rates, and these are reflected in distributions of plasma PK parameters across a population. Additionally, granuloma structural heterogeneity (including differences in size and composition) can lead to differences in antibiotic exposure at the lesion level. To capture both sources of heterogeneity, which occur at different length scales, we devised a sequential calibration scheme to calibrate the PK model from data (Figure 3; parameters in Tables 2 and 3).

TABLE 4 | Pharmacodynamic parameter and sources for data used for parameter fitting/estimation. Units of 1/timestep represent per model timestep of 10 min.

\begin{tabular}{|c|c|c|c|c|c|}
\hline Parameter & INH & RIF & EMB & PZA & Sources \\
\hline Intracellular $\mathrm{C}_{50}(\mathrm{mg} / \mathrm{L})$ & 0.070 & 20 & 5.22 & 70 & (Jayaram et al., 2003; Jayaram et al., 2004; Hartkoorn et al., 2007; Sarathy et al., 2018) \\
\hline Extracellular, replicating $\mathrm{C}_{50}(\mathrm{mg} / \mathrm{L})$ & 0.015 & 1.23 & 0.05 & 370 & (Jayaram et al., 2003; Jayaram et al., 2004; Hartkoorn et al., 2007; Sarathy et al., 2018) \\
\hline $\begin{array}{l}\text { Extracellular, } \\
\text { Non-replicating } \mathrm{C}_{50}(\mathrm{mg} / \mathrm{L})\end{array}$ & 17.7 & 81 & 1000 & 370 & (Lakshminarayana et al., 2015; Sarathy et al., 2018) \\
\hline Intracellular Emax (1/timestep) & 0.0056 & 0.014 & 0.026 & 0.0006 & (Jayaram et al., 2003; Jayaram et al., 2004; Hartkoorn et al., 2007; Sarathy et al., 2018) \\
\hline Extracellular Emax (1/timestep) & 0.0056 & 0.019 & 0.025 & 0.007 & (Jayaram et al., 2003; Jayaram et al., 2004; Hartkoorn et al., 2007; Sarathy et al., 2018) \\
\hline Intracellular hill constant, $\mathrm{h}$ & 1 & 0.5 & 2.5 & 3.2 & (Jayaram et al., 2003; Jayaram et al., 2004; Hartkoorn et al., 2007; Sarathy et al., 2018) \\
\hline Extracellular hill constant, $\mathrm{h}$ & 1 & 0.5 & 1.5 & 1 & (Jayaram et al., 2003; Jayaram et al., 2004; Hartkoorn et al., 2007; Sarathy et al., 2018) \\
\hline
\end{tabular}


Figure 4 shows antibiotic total concentrations (sum of free and bound) within both plasma and granulomas for all four firstline antibiotics (INH, RIF, EMB, and PZA). Results are shown for $24 \mathrm{~h}$ following an oral dose and compared to experimental data. Using our sequential calibration scheme, we capture a large proportion of the experimentally observed antibiotic concentration data in both plasma and granulomas. The $\mathrm{C}_{50}$ values for each bacterial subpopulation, obtained by fitting the data referenced in Table 3, indicate the free-drug concentration when a given antibiotic is at half its maximum bacterial killing rate. INH, RIF, and EMB achieve sufficient concentrations to kill extracellular replicating Mtb for a majority of the dosing period. Both INH and EMB can achieve concentrations above the $\mathrm{C}_{50}$ for intracellular Mtb. Only RIF approaches concentrations necessary to achieve bactericidal activity against non-replicating Mtb. Based on average granuloma concentrations, PZA appears to have little sterilizing activity in granulomas.

Figure 4 also shows antibiotic total concentrations as a function of position throughout the grid, at the time of maximal average granuloma drug concentration following a single oral dose of each antibiotic in the same in silico granuloma, shown in grayscale to allow for the illustration of gradual concentration changes. INH shows a relatively homogenous distribution in the lesion that rapidly clears as INH is eliminated in the plasma. RIF accumulates poorly in granulomas at early time points but can slowly accumulate in the caseum following multiple doses (Supplementary Figure 2). EMB tends to accumulate in regions with a high density of macrophages but fails to diffuse into caseum significantly. PZA shows a slight accumulation in caseum relative to the macrophage-rich regions of the granuloma. To further validate our model, Figure 5 shows PZA distribution identified experimentally using matrix-assisted laser desorption/ ionization mass spectrometry imaging (MALDI-MSI) as we have done previously (Prideaux et al., 2015) and compares the PZA signal intensity distribution to two simulated granulomas. Overall, our simulated distributions for other antibiotics agree with observations made through MALDI-MSI in TB granulomas (Prideaux et al., 2015; Zimmerman et al., 2017). These qualitative features observed in the simulations for each antibiotic were not used in calibrating the tissue PK parameters, but rather resulted from estimating and fitting the tissue PK parameters to average granulomas concentrations (Table 1).

\section{Single-Drug Treatments Sterilize Granulomas at Different Rates and to Different Extents}

We next tested the abilities of each first-line antibiotic, when dosed alone, to sterilize granulomas with average plasma PK exposure and low or high-CFU (Groups 1 and 2 of Figure 3). The rates and extents of sterilization differ for each antibiotic in low-CFU granulomas, as shown in Figure 6A, due to differences
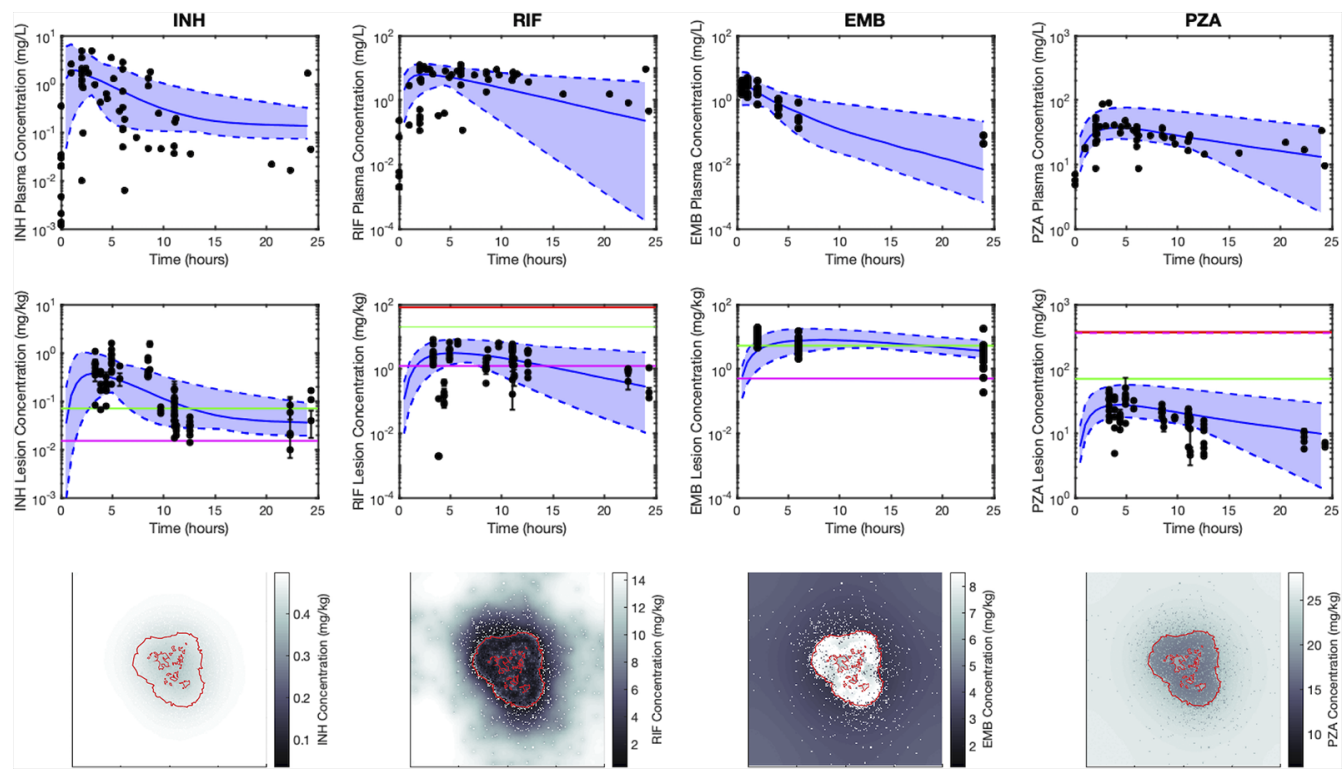

FIGURE 4 | Simulations capture both the experimentally observed temporal and spatial antibiotic concentrations. Simulations and data for each antibiotic [isoniazid $(\mathrm{INH})$, rifampin (RIF), ethambutol (EMB), and pyrazinamide (PZA)], dosed singly, are shown in different columns, respectively. The top row shows plasma concentrations and the middle row shows average lesion concentrations with varying plasma pharmacokinetic (PK) parameters (median, solid blue line; range between minimum and maximum of simulations, blue shade) and experimentally measured antibiotic concentrations (black points). Concentrations in granulomas are in $\mathrm{mg} / \mathrm{kg}$ (assuming tissue density is approximately $1 \mathrm{~kg} / \mathrm{L}$ ), and reflect the sum of concentrations of free, bound and intracellular drug. Horizontal lines represent the $\mathrm{C}_{50}$ values for intracellular (green), extracellular replicating (magenta) and non-replicating (red) subpopulations of Mtb ( $\mathrm{C}_{50}$ values not shown are above the range of lesion concentrations displayed on the plot). Data in the middle row are measurements from human granulomas (INH, RIF, and PZA (Prideaux et al., 2015)) and rabbit granulomas [EMB (Zimmerman et al., 2017)]. The bottom row shows spatial distribution of antibiotics in GranSim at the time of the maximal average lesion concentration. Red outlines indicate edge of granuloma (outer line) and caseated locations (inner lines). 
A

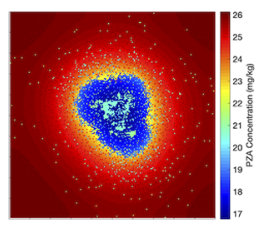

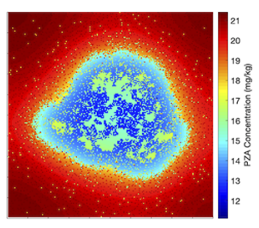

B Subject \#1
$5 \mathrm{~h}$
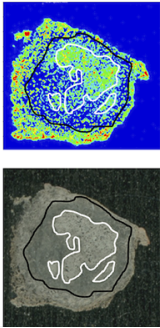

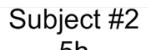
$5 \mathrm{~h}$
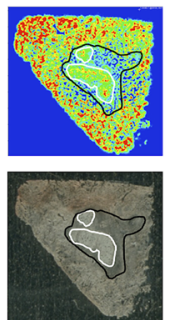

Subject \#1

$5 \mathrm{~h}$

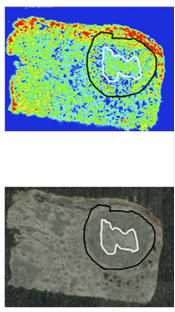

FIGURE 5 | Comparison of spatial distribution of pyrazinamide (PZA) in GranSim (A) and in experimental images of granulomas using matrix-assisted laser desorption/ionization mass spectrometry imaging (MALDI-MSI) (B). The simulation images show heat maps of the spatial distribution of PZA at $5 \mathrm{~h}$ after a single-PZA dose. In the simulated concentration heat maps, shown in color to mimic the images from MALDI-MSI (A), the red area corresponds to lung tissue outside of the granuloma, the darker blue regions indicates regions inside the granuloma with higher densities of macrophages, and the lighter blue to green sections show correspond to caseated regions. Both simulation images are on a 200 by 200 grid, representing a $4 \mathrm{~mm}$ by $4 \mathrm{~mm}$ section of lung tissue. Experimental images (B) show PZA distribution in granulomas imaged with MALDI-MSI, with granuloma boundary outlined in black, and caseated regions outlined in white. Both simulation and experiments show some accumulation of PZA inside caseous regions, relative to the cellular portions of the granuloma.

in sterilizing activity against various subpopulations of bacteria as well as the antibiotic distribution within granulomas. After 180 days of treatment, single-drug therapy with RIF sterilizes all low-CFU granulomas. INH sterilized 93\% low-CFU granulomas. EMB and PZA each sterilize just $32 \%$ and $1.7 \%$ of low-CFU granulomas, respectively. INH and EMB all have early sterilizing ability and were able to sterilize $29 \%$ and $31 \%$ of granulomas after two weeks, respectively. RIF alone only sterilized $5 \%$ by 2 weeks, and PZA failed to sterilize any granulomas by two weeks.

Our simulations show that INH can quickly distribute within granulomas, and in sufficient concentrations to kill both intracellular and extracellular replicating bacteria, and therefore
A
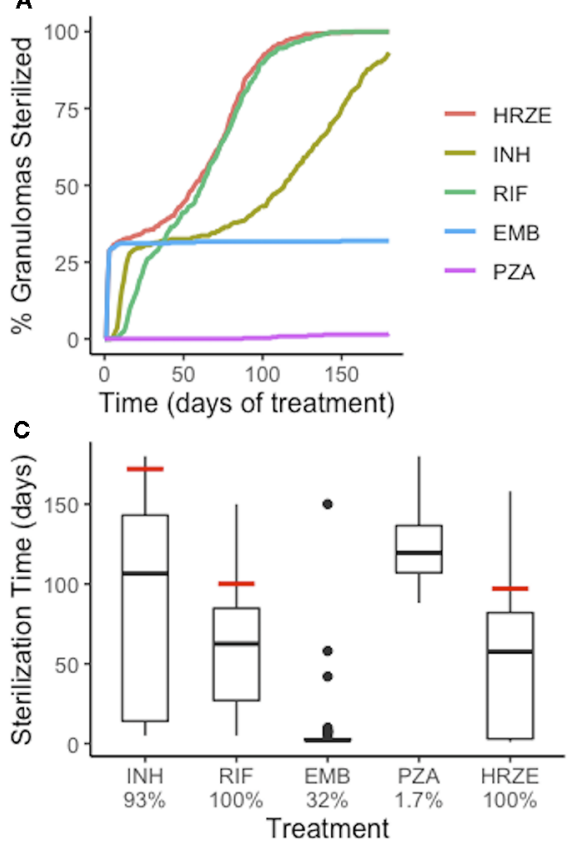

B
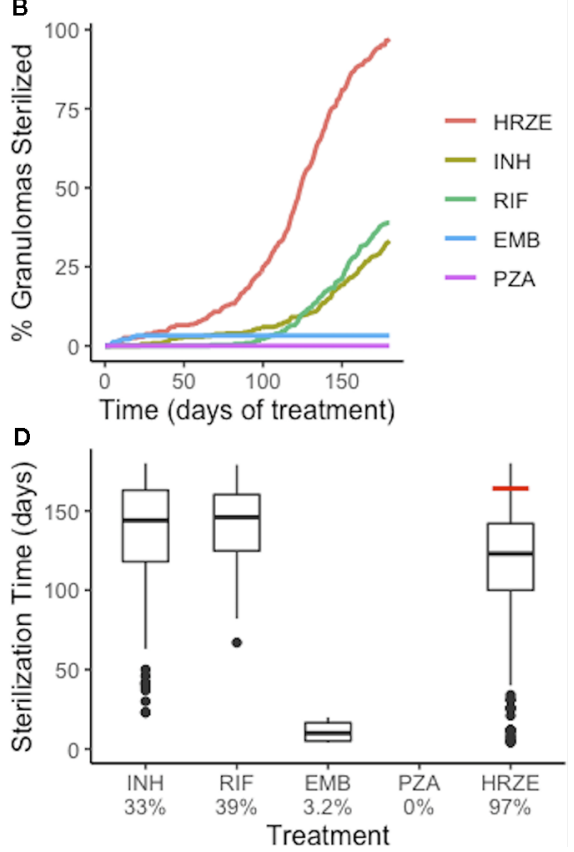

FIGURE 6 | Single-antibiotic treatments and combination therapy of low-CFU (A, C) and high-CFU (B, D) granulomas show different sterilizing rates and extents for each of the first-line antibiotics and all four antibiotics together (HRZE). (A, B) show the percentage of granulomas sterilized over the course of treatment for both groups of granulomas. (C, D) show the distribution of sterilization times for only the granulomas that sterilized for each treatment, with the time when $90 \%$ of granulomas were sterilized indicated by a red line. Percentage below each treatment indicates the total percentage of granulomas that sterilized. For example, EMB sterilized 32\% of low-CFU granulomas (C), and of those sterilized granulomas, a majority of them sterilized in the first few days (indicated by the box plot collapsing to a line). 
provides rapid sterilization for some granulomas, as shown in Figures 4 and 5. However, with poor sterilizing ability against non-replicating Mtb found in caseum (Sarathy et al., 2018), INH usually requires many months to sterilize granulomas that have a high number of non-replicating Mtb and leads to the drawn-out sterilization of non-replicating Mtb in INH-treated granulomas. EMB, similar to INH, has poor ability to kill non-replicating Mtb, so our simulations show it is only able to sterilize a subset of granulomas, even though it distributes throughout cellular regions of the granuloma. However, it does rapidly kill both extracellular replicating and intracellular $\mathrm{Mtb}$, indicated by the percentage of granulomas sterilized by two weeks, which is consistent with favorable early bactericidal activity (EBA) for EMB (Donald and Diacon, 2008). Because INH and EMB are bacteriostatic and have low ability to kill non-replicating Mtb, sterilization time (for INH) and total Mtb remaining in the granuloma (for EMB) are highly correlated with the initial number of non-replicating bacteria present in the granuloma (Supplementary Figure 3). RIF shows more complete sterilization of low-CFU granulomas than any other individual antibiotic as it has some sterilizing ability against each subpopulation of bacteria.

We observe similar trends with single-drug treatments in high-CFU granulomas (Figure 6B). Overall, the sterilization times are longer when compared to low-CFU granulomas. INH and RIF both sterilize lower percentages of the high-CFU granulomas than they do low-CFU granulomas. High-CFU granulomas are also more likely to have higher total numbers of non-replicating $\mathrm{Mtb}$, decreasing the ability of $\mathrm{INH}$ to completely sterilize these granulomas. RIF, with weakened ability to kill intracellular Mtb due to low granuloma concentrations, fails to kill all intracellular $\mathrm{Mtb}$ in some granulomas. This weakness is amplified in larger granulomas, slowing diffusion of antibiotics into the granulomas.

\section{Specialization of Individual Antibiotics Contributes to Success of Combination Therapy}

Combination therapy-all four first-line antibiotics-sterilizes low-CFU granulomas at nearly the same rate as the best singleantibiotic treatment (RIF) (Figure 6). All granulomas are sterilized after 147 days of combination therapy, with 33\% sterilized after 2 weeks. The difference in early versus late sterilizing ability for the single-drug treatments is one reason why the combination therapy shows faster and more complete sterilization than any one drug on its own. Early in treatment, INH and EMB do much of the killing, and the presence of RIF completes the sterilization.

The benefit of combination therapy is more dramatic for high-CFU granulomas (Group 2 of Figure 3). Here, treatment with INH or RIF show only $33 \%$ and $39 \%$ sterilization after 180 days of therapy, respectively, compared to $97 \%$ of granulomas sterilized with HRZE (Figure 6D). Although RIF is able to sterilize granulomas as well as HRZE in low-CFU granulomas, the same behavior is not observed in high-CFU granulomas. RIF is relatively slow at killing intracellular bacteria. In the low-CFU granulomas, the number of intracellular $\mathrm{Mtb}$ is low enough where RIF can kill these bacteria eventually. In high-CFU granulomas, RIF is not always able to kill intracellular Mtb fast enough to keep up with its replication, and therefore fails to sterilize all high-CFU granulomas. The presence of INH and EMB provide assistance in killing the intracellular Mtb, so the combination of antibiotics allows for more complete sterilization. Our model predicts that the different abilities to kill each of the subpopulations of bacteria and the different distributions within granulomas complement each other in combination therapy.

During combination therapy (HRZE), a majority of bacterial death is due to antibiotics; antibiotics are responsible for roughly an order of magnitude more bacterial death than the immune response, and two orders more than bacterial death in caseum representing a lack of oxygen and nutrients (Supplementary Figure 4). This trend is consistent across the single-drug treatments with the exception of PZA, which shows the poorest efficacy and thus allows for continued bacteria growth and continued slow killing via the immune response.

\section{High-CFU and Low PK Exposure Lengthen Sterilization Times During Combination Therapy}

We next tested how sterilization time and thus the necessary length of treatment is affected by plasma PK variability between individuals and granuloma heterogeneity. We compared the sterilization times of all four granuloma groups (Figure 3B) when treated with daily doses of HRZE. Figure 7A shows the distribution of sterilization times for each of these treatment scenarios. Simulating the low-CFU granulomas with low PK exposure (Group 2) results in a shift in the distribution towards longer sterilization times relative to average PK exposure (Group 1), with the $90 \%$ sterilization time increasing from 97 to 133 days. In contrast, 165 days of HRZE are required to sterilize $90 \%$ of the high-CFU granulomas with average PK exposure, and 90\% sterilization cannot be reached within 6 months of treatment when those same granulomas have low PK exposure.

With some granulomas failing to sterilize after 180 days of treatment, we sought to analyze the characteristics of those granulomas. We grouped our granulomas into four different "risk" categories: low (sterilize in under 90 days of HRZE), medium (sterilize between 90 and 150 days), high (sterilize after 150 days), and unsterilized. For each of these groups, we compared characteristics of the granulomas before treatment to see what types of granulomas have different levels of risk. Unsterilized and high-risk granulomas tend to be higher in CFU, size, and amount of caseation (Supplementary Figure 5), with median CFU/granuloma levels before treatment of $1.1 \times 10^{5}, 6.0 \times 10^{4}, 2.1 \times 10^{4}$, and $1 \times 10^{3}$ for the unsterilized, high, medium, and low risk categories. However, these pretreatment characteristics are not sufficient in predicting whether a specific granuloma will fail to sterilize during treatment, as there are some granulomas with high CFU, diameter, and caseation that sterilize within 90 days. Although these low risk granulomas look like high risk or unsterilized granulomas, they have higher percentages of intracellular Mtb. At the beginning of treatment 

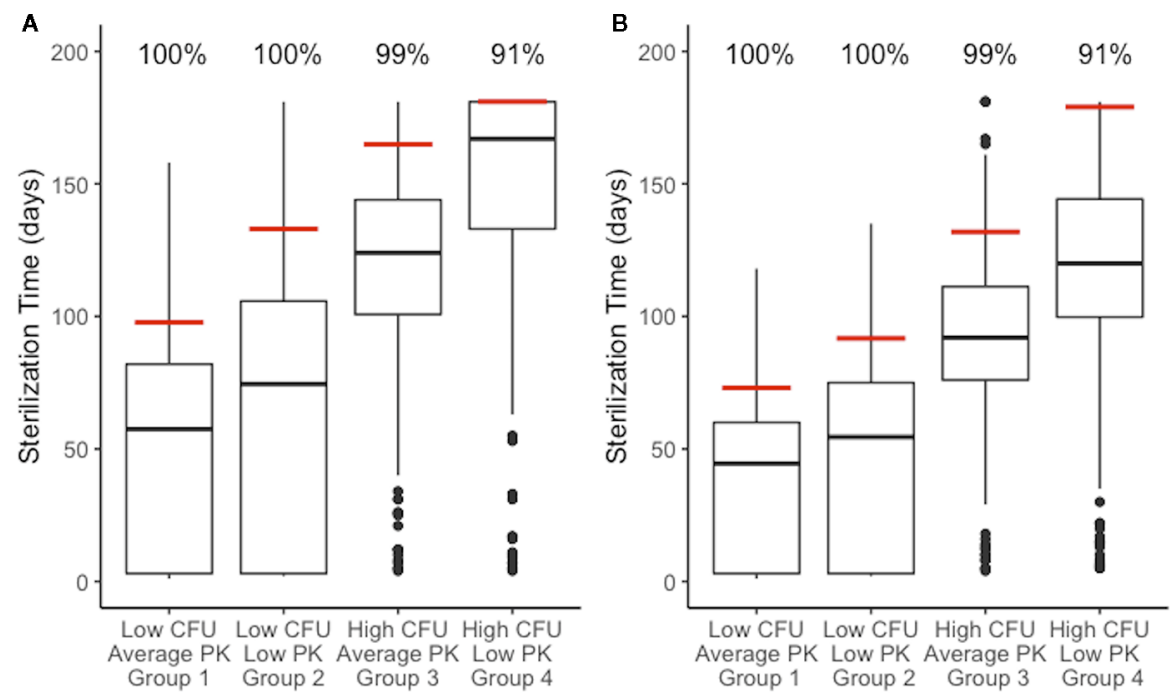

FIGURE 7 | Distributions of sterilization times for different granuloma treatment groups, referenced in Figure 3B, treated with HRZE indicate factors that negatively impact sterilization. (A) shows simulations of the standard regimen (HRZE). (B) shows the simulations of high rifampin (RIF) dose treatments (20 mg/kg). Each boxplot shows the sterilization time distribution of a treatment group, with outlying simulations as dots and the red line indicating the time of $99 \%$ sterilization.. Low CFU granulomas with average pharmacokinetic (PK) exposure sterilize the fastest. Low CFU granulomas with low PK exposure show a shift to longer sterilization times compared with average exposure. Similarly, high CFU granulomas with average exposure sterilize faster than high CFU granulomas with low exposure. Results for low CFU and high CFU with average PK are shown in Figure 6 and are plotted again here for comparison.

with HRZE, these intracellular bacteria can be quickly killed, making the granulomas easier to sterilize.

Variation in plasma PK exposure may impact treatment with some antibiotics more profoundly than others. To test this, we sampled a set of 200 plasma PK parameters from the ranges used in calibrating the PK model (Table 2). With each of these plasma PK parameter sets that generate different levels of exposure in plasma, we treated the same granuloma with each single-drug treatment (Figure 8). Overall, RIF is most impacted by natural variability in plasma exposure, and varying plasma PK parameters for RIF results in a wider spread of treatment outcomes than other antibiotics, ranging from a minimum sterilization time of 38 days to unsterilized granulomas by the end of treatment (Figure 8). This indicates that optimizing dose for RIF and other antibiotics that are particularly sensitive to variations in PK existing in human populations may be critical in designing better regimens.

\section{Treatment Time Can Be Shortened for Some Granulomas by Increasing the Dose of RIF}

There have been numerous efforts to shorten TB treatment regimens and clinical trials that involve replacing one or more antibiotics in the standard regimen or increasing doses of the firstline antibiotics (Gillespie et al., 2014; Jindani et al., 2014). Increasing RIF dosage to $20 \mathrm{mg} / \mathrm{kg}$ is a strategy applied in several clinical trials (Diacon et al., 2007; Boeree et al., 2017; Peloquin et al., 2017), and is rational because it could lessen the impact $\mathrm{PK}$ variability has on RIF given our results (Figure 7). We investigated how increasing the RIF dose impacts granuloma sterilization time while accounting for granuloma heterogeneity and PK variability. To simulate high RIF dose treatments, we simulated each treatment group of granulomas with the same combination regimen as before but increased the RIF dose to $20 \mathrm{mg} / \mathrm{kg}$.

Increasing the RIF dose in combination therapy results in shorter average sterilization times as compared to the standard combination therapy (Figure 7B). The 90\% sterilization times for low-CFU granulomas decrease by 25 days for average PK and 41 days for low PK exposure. High-CFU granulomas with average PK exposure showed a decrease in $90 \%$ sterilization times by 33 days when treated with a high RIF dose, and the low PK exposure simulations increased the percent of sterilized granulomas from $62 \%$ to $91 \%$ (the latter giving a $90 \%$ sterilization time of 179 days). Overall, the improvement observed is greater in the low PK exposure simulations than in average PK simulations.

\section{DISCUSSION}

Treatment of drug-susceptible $\mathrm{TB}$ requires multiple months of antibiotics, after which treatment may still fail due to unsterilized granulomas. A better understanding of the first-line combination therapy, HRZE, will help to develop rational approaches to reduce treatment duration and improve cure rates. To analyze the use of first-line antibiotics and the factors that impact granuloma sterilization and conditions of treatment failure, we developed a computational framework that captures both granuloma heterogeneity and PK variability observed in human studies to determine the rate and extent of sterilization during treatment with first-line TB antibiotics at the granuloma scale.

To place the findings of our work into better context with clinical evidence that has been gathered on first-line TB 

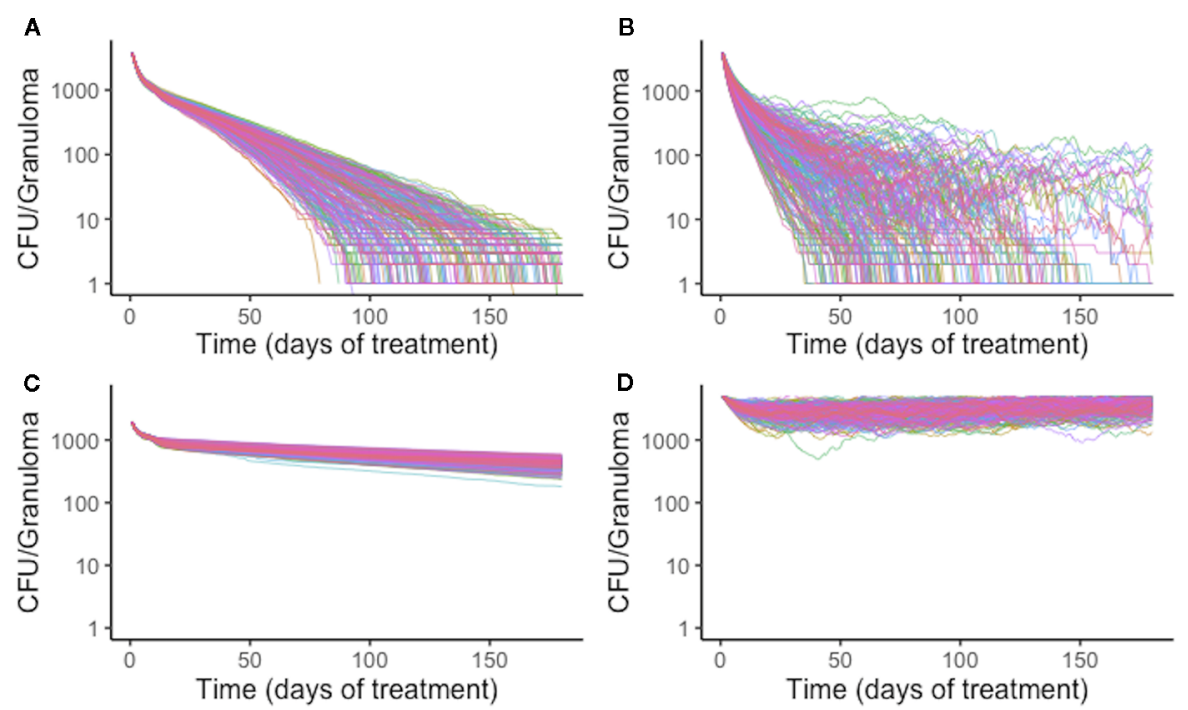

FIGURE 8 | Simulation treatment outcomes of single-drug treatments of the same in silico granuloma vary with different plasma pharmacokinetic (PK) parameter sets. A single granuloma was treated with each of the single-drug treatments with 200 different plasma PK parameter sets. Above shows the CFU for each granuloma simulation over time during treatment for isoniazid (INH) (A), rifampin (RIF) (B), ethambutol (EMB) (C), and pyrazinamide (PZA) (D). The standard deviation of sterilization times for different plasma PK parameter sets for RIF normalized to mean sterilization time is 0.40 . This indicates greater variability in sterilization times due to changes in plasma PK for RIF compared to INH, for which the value is 0.19 . EMB and PZA have standard deviations of log-transformed CFU normalized to the mean at the end of treatment standard deviations of 0.033 and 0.034 , respectively.

antibiotics, we compared our simulations for single-drug treatments and combination treatments to early bactericidal activities (EBA) measured in multiple studies (Table 5). The EBA estimates based on the simulations are shown as the decrease in $\log _{10}(\mathrm{CFU}) /$ day for each treatment. Many of our simulated estimates are near the clinically measured EBA values (given as reported ranges or confidence intervals). For simulated EBA estimates that do not match clinical results, our simulations tend to predict lower EBA values than those observed clinically. Our EBA estimates account for the entire granuloma's CFU count, and it is possible that we predict lower EBAs as our simulations detect more remaining bacteria than those that would be detected clinically in sputum due to limitations of detection in assays used.

We found that typical PK variability and granuloma heterogeneity can create scenarios that profoundly impact sterilization rates and treatment success. The level of antibiotic concentration in plasma leads to commensurate concentrations within granulomas, creating differences in sterilization rates. Individuals with lower plasma PK exposure are at higher risk

TABLE 5 | Comparison of antibiotic treatment simulations to clinical early bactericidal activity (EBA) data. Table shows the simulation EBA, calculated as the decrease in $\log _{10}$ (CFU) per day over the day intervals indicted. Values reported are the mean daily decrease in CFU over all granulomas simulated with the standard regimen doses and average PK. Standard deviation is indicated in parenthesis. The clinical EBA values reported are taken from a number of studies and reviews. The simulation EBA for $(0-x)$ days is calculated as $\left(\log _{10}(C F U\right.$ day 0$\left.)-\log _{10}\right)(C F U$ day $\left.x)\right) / x$.

\begin{tabular}{|c|c|c|c|c|c|c|}
\hline \multirow[b]{2}{*}{ Antibiotic } & \multicolumn{3}{|c|}{ Simulation, Mean (SD) } & \multicolumn{3}{|c|}{ Clinical } \\
\hline & EBA & EBA & EBA & EBA 0-2 Days & EBA 0-5 Days & EBA 0-14 Days \\
\hline & $0-2$ & $0-5$ & $0-14$ & & & \\
\hline & Days & Days & Days & & & \\
\hline $\mathrm{INH}$ & $\begin{array}{l}0.16 \\
(0.062)\end{array}$ & $\begin{array}{l}0.13 \\
(0.066)\end{array}$ & $\begin{array}{l}0.079 \\
(0.051)\end{array}$ & $\begin{array}{l}\text { Ranges from 0.37-0.77 involving } 13 \text { studies } \\
\text { summarized in (Donald and Diacon, 2008) }\end{array}$ & $\begin{array}{l}0.25 \text { (range of 0.19-0.40) as } \\
\text { summarized in (Donald and Diacon, } \\
2008 \text { ) }\end{array}$ & $\begin{array}{l}\text { Ranges from 0.189-0.192 involving two } \\
\text { studies summarized in (Donald and Diacon, } \\
\text { 2008) }\end{array}$ \\
\hline RIF & $\begin{array}{l}0.15 \\
(0.044)\end{array}$ & $\begin{array}{l}0.12 \\
(0.037)\end{array}$ & $\begin{array}{l}0.086 \\
(0.027)\end{array}$ & $\begin{array}{l}\text { Ranges 0.174-0.631 involving } 8 \text { studies } \\
\text { summarized in (Donald and Diacon, 2008) }\end{array}$ & $\begin{array}{l}0.226 \text { (SD 0.144) reported in (Sirgel } \\
\text { et al., 2005) }\end{array}$ & $\begin{array}{l}{ }^{b} 0.11 \text { (SD 0.096) reported in (Donald and } \\
\text { Diacon, 2008) from (Jindani et al., 1980) }\end{array}$ \\
\hline $\mathrm{EMB}$ & $\begin{array}{l}0.45 \\
(0.36)\end{array}$ & $\begin{array}{l}0.20 \\
(0.16)\end{array}$ & $\begin{array}{l}0.082 \\
(0.061)\end{array}$ & $\begin{array}{l}0.25 \text { (95\% Cl: 0.06-0.45) pooled in } \\
\text { (Bonnett et al., 2017) }\end{array}$ & NA & $\begin{array}{l}{ }^{b} 0.16 \text { (SD 0.090) reported in (Jindani et al., } \\
\text { 2003) }\end{array}$ \\
\hline PZA & $\begin{array}{l}0.014 \\
(0.009)\end{array}$ & $\begin{array}{l}0.014 \\
(0.007)\end{array}$ & $\begin{array}{l}0.012 \\
(0.006)\end{array}$ & $\begin{array}{l}0.01 \text { (95\% Cl: }-0.07-0.09) \text { pooled in } \\
\text { (Bonnett et al., 2017) }\end{array}$ & NA & $\begin{array}{l}{ }^{b} 0.11 \text { (SD 0.038) reported in (Jindani et al., } \\
2003 \text { ) }\end{array}$ \\
\hline HRZE & $\begin{array}{l}0.49 \\
(0.34)\end{array}$ & $\begin{array}{l}0.24 \\
(0.15)\end{array}$ & $\begin{array}{l}0.11 \\
(0.052)\end{array}$ & $\begin{array}{l}0.3 \text { (95\% Cl: 0.09-0.50) pooled in (Bonnett } \\
\text { et al., 2017) }\end{array}$ & $\begin{array}{l}{ }^{\mathrm{a}} 0.16 \text { (95\% Cl: 0.09-0.24) pooled in } \\
\text { (Bonnett et al., 2017) }\end{array}$ & $\begin{array}{l}0.16 \text { (95\% Cl: 0.11-0.21) pooled in (Bonnett } \\
\text { et al., 2017) }\end{array}$ \\
\hline
\end{tabular}

${ }^{a} E B A$ 0-7 Days

${ }^{b}$ EBA 2-14 Days 
of antibiotic underexposure in selected granulomas. When coupled with complex and caseous granuloma structure with impaired vascular supply, this can lead to longer sterilization times using standard HRZE TB therapy (Figure 7). Other models using various experimental data, including hollow fiber experiments, show that low drug exposure can lead to decreased rates in bacterial killing (Srivastava and Gumbo, 2011), and have used variability in PK to predict variability in required treatment durations (Magombedze et al., 2018). The model we present builds on these findings by providing the ability to simulate sterilization in a granuloma, while accounting for human-based PK variability and granuloma structure. The benefit of simulating treatment in the context of the whole granuloma is that it includes the spatial microenvironments that can influence both antibiotic distribution and bacterial susceptibility or tolerance to antibiotics. Treating each Mtb as an individual agent also provides the ability to simulate treatment while accounting specifically for antibiotic resistance (Pienaar et al., 2018). Our model is a tool that can provide quantitative predictions and sterilization times for a given regimen at a granuloma level, the possibility to predict entire host treatment through linking of plasma pharmacokinetics, and the potential to search for optimal treatment regimens (Cicchese et al., 2017).

We show that treatment with any of the current first-line TB antibiotics alone is not sufficient to sterilize all granulomas, and that combinations of antibiotics result in more rapid and complete sterilization. Although RIF shows the best sterilizing ability on its own and is about as effective as HRZE in low-CFU granulomas, RIF alone fails to sterilize many of the high-CFU granulomas, where it only sterilizes $39 \%$ of granulomas compared to $97 \%$ with HRZE. Although our simulations predict that PZA sterilizes very few granulomas on its own, evidence suggests that PZA does show sterilizing ability when administered on its own, and suggests that our simulations underestimate its activity and that there is discrepancy between the in vitro activity of PZA and in vivo efficacy that our model does not capture (Irwin et al., 2016; Lanoix et al., 2016; Blanc et al., 2018).

Granulomas with increased CFU and lower antibiotic exposure can dramatically increase sterilization time and increase the risk of granulomas that do not completely sterilize. Granulomas with high risk of not sterilizing tend to be larger and have more CFU; however, the type of bacteria present in those granulomas may affect the risk of treatment failure as well. Granulomas with high CFU may still have a low risk of treatment failure if they have high percentages of intracellular Mtb. Because some of the antibiotics in HRZE are good at quickly killing this subpopulation, these granulomas that look like high risk granulomas pretreatment, quickly become low risk granulomas as treatment begins.

RIF is the antibiotic that provides the best sterilizing ability on its own, but also is the antibiotic that shows the highest interindividual PK variability (Stott et al., 2018) and is most impacted by $\mathrm{PK}$ variability. To reduce the impact the sensitivity RIF has to PK variability, we simulated HRZE treatment while doubling the RIF dose. Indeed, we did observe faster granuloma sterilization and more complete sterilization in high-CFU, low PK granulomas, yet some granulomas in that group still failed to sterilize. Additionally, there was only a slight improvement in sterilization times for granulomas that were already easy to treat, indicating there might only be a modest improvement in treatment for a subset of those granulomas. Understanding an individual's PK profile for different drugs would be an important step in developing a personalized medicine approach to treatment.

While our model can recapitulate key experimental observations and also predict TB treatment outcomes, there are several limitations to our findings. Clinical results measure outcomes at the host level, and GranSim fundamentally simulates treatment and sterilization at the granuloma scale. The relevance of our results relies on the assumption that treatment at the granuloma scale is indicative of treatment at a host scale. Our model simulates primary granulomas and does not fully capture the full complexity of multiple pulmonary lesions as is observed during TB disease. It is appreciated that non-replicating and persisting $\mathrm{Mtb}$ are critical targets to achieve full sterilization of lesions, and while we observe this in our model, their importance could be amplified in cavitary disease or fibrotic lesions that are not captured in our model. Further, directly relating in vitro antimicrobial activity to in vivo efficacy does not necessarily capture the full range of antimicrobial activity that occurs within granulomas and may partially account for any discrepancies between our simulation results and clinical observations. An additional limitation of our model is that it currently assumes there are no interactions occurring between antibiotics, and synergistic or antagonistic combinations may be relevant in determining regimen efficacy (Swaminathan et al., 2016; Ma et al., 2019). Going forward, we are currently introducing synergistic and antagonistic antibiotic interactions to improve the PD model and further refine our estimates and predictions of granuloma sterilization (Chandrasekaran et al., 2016; Cokol et al., 2017; Cokol et al., 2018). The current model also does not include the development of antibacterial resistance, which may profoundly impact granuloma sterilization; see (Pienaar et al., 2018) for a previously published model examining development of resistance and a discussion of modeling resistance development. Finally, this work drew on data sets from a variety of human and animal studies, and predictions of treatment efficacy for other and newer drugs is dependent on the acquisition of similar data sets.

The significant impact that population PK variability and granuloma heterogeneity have on granuloma sterilization highlights the continued need for new approaches and drugs for treatment, and optimization of new regimens. Close collaboration between wet lab and computational scientists will help facilitate the evaluation of these new approaches and provide a more efficient and comprehensive development of new ways to treat TB.

\section{DATA AVAILABILITY STATEMENT}

The datasets generated for this study are available on request to the corresponding author. 


\section{AUTHOR CONTRIBUTIONS}

The modeling and simulations of this work were completed by JC. VD provided assistance in pharmacokinetic and pharmacodynamic modeling, as well as MALDI-MSI. All authors (JC, VD, DK, and JL) contributed to the conceptualization of this work, analysis of results, and writing and editing.

\section{FUNDING}

This research was supported by the following grants from the National Institutes of Health: U01HL131072 (to DK, JL, and VD), R01AI123093 (to DK), R01AI106398, and 1S10OD018072-01A1 (to VD). Simulations used resources of the National Energy Research Scientific Computing Center, which is supported by the

\section{REFERENCES}

Bhusal, Y., Shiohira, C. M., and Yamane, N. (2005). Determination of in vitro synergy when three antimicrobial agents are combined against Mycobacterium tuberculosis. Int. J. Antimicrob. Agents 26, 292-297. doi: 10.1016/j.jjantimicag.2005.05.005

Blanc, L., Sarathy, J. P., Alvarez Cabrera, N., O'Brien, P., Dias-Freedman, I., Mina, M., et al. (2018). Impact of immunopathology on the antituberculous activity of pyrazinamide. J. Exp. Med. 215, 1975-1986. doi: 10.1084/jem.20180518

Blum, M., Demierre, A., Grant, D. M., Heim, M., and Meyer, U. A. (1991). Molecular mechanism of slow acetylation of drugs and carcinogens in humans. Proc. Natl. Acad. Sci. 88, 5237-5241. doi: 10.1073/pnas.88.12.5237

Boeree, M. J., Heinrich, N., Aarnoutse, R., Diacon, A. H., Dawson, R., Rehal, S., et al. (2017). High-dose rifampicin, moxifloxacin, and SQ109 for treating tuberculosis: a multi-arm, multi-stage randomised controlled trial. Lancet Infect. Dis. 17, 39-49. doi: 10.1016/S1473-3099(16)30274-2

Bonnett, L. J., Ken-dror, G., Koh, G. C. K. W., and Davies, G. R. (2017). Comparing the Efficacy of Drug Regimens for Pulmonary Tuberculosis: Meta-analysis of Endpoints in Early-Phase Clinical Trials. Clin. Infect. Dis. 65, 46-54. doi: 10.1093/cid/cix247

Chandrasekaran, S., Cokol-Cakmak, M., Sahin, N., Kazan, H., Yilancioglu, K., Collins, J. J., et al. (2016). Chemogenomics and orthology-based design of antibiotic combination therapies. Mol. Syst. Biol. 12, 872. doi: 10.15252/msb.20156777

Cicchese, J. M., Pienaar, E., Kirschner, D. E., and Linderman, J. J. (2017). Applying Optimization Algorithms to Tuberculosis Antibiotic Treatment Regimens. Cell. Mol. Bioeng. 10, 523-535. doi: 10.1007/s12195-017-0507-6

Cilfone, N. A., Perry, C. R., Kirschner, D. E., and Linderman, J. J. (2013). Multiscale modeling predicts a balance of tumor necrosis factor-a and interleukin-10 controls the granuloma environment during Mycobacterium truberculosis infection. PloS One 8, e68680. doi: 10.1371/journal.pone.0068680

Cilfone, N. A., Kirschner, D. E., and Linderman, J. J. (2015). Strategies for Efficient Numerical Implementation of Hybrid Multi-scale Agent-Based Models to Describe Biological Systems. Cell. Mol. Bioeng. 8, 119-136. doi: 10.1007/ s12195-014-0363-6

Cokol, M., Kuru, N., Bicak, E., Larkins-Ford, J., and Aldridge, B. B. (2017). Efficient measurement and factorization of high-order drug interactions in Mycobacterium tuberculosis. Sci. Adv. 3, e1701881. doi: 10.1126/sciadv.1701881

Cokol, M., Li, C., and Chandrasekaran, S. (2018). Chemogenomic model identifies synergistic drug combinations robust to the pathogen microenvironment. PloS Comput. Biol. 14, 1-24. doi: 10.1371/journal.pcbi.1006677

Dartois, V. (2014). The path of anti-tuberculosis drugs: from blood to lesions to mycobacterial cells. Nat. Rev. Microbiol. 12, 159-167. doi: 10.1038/ nrmicro3200

Denti, P., Jeremiah, K., Chigutsa, E., Faurholt-Jepsen, D., PrayGod, G., Range, N., et al. (2015). Pharmacokinetics of isoniazid, pyrazinamide, and ethambutol in newly diagnosed pulmonary TB patients in Tanzania. PloS One 10, 1-19. doi: 10.1371/journal.pone.0141002
Office of Science of the U.S. Department of Energy under Contract No. ACI-1053575 and the Extreme Science and Engineering Discovery Environment (XSEDE), which is supported by the National Science Foundation grant MCB140228.

\section{ACKNOWLEDGMENTS}

We thank Paul Wolberg for computational assistance and support.

\section{SUPPLEMENTARY MATERIAL}

The Supplementary Material for this article can be found online at: https://www.frontiersin.org/articles/10.3389/fphar.2020.00333/ full\#supplementary-material

Diacon, A. H., Patientia, R. F., Venter, A., Van Helden, P. D., Smith, P. J., Mcilleron, H., et al. (2007). Early Bactericidal Activity of High-Dose Rifampin in Patients with Pulmonary Tuberculosis Evidenced by Positive Sputum Smears. Antimicrob. Agents Chemother. 51, 2994-2996. doi: 10.1128/AAC.01474-06

Donald, P. R., and Diacon, A. H. (2008). The early bactericidal activity of antituberculosis drugs: a literature review. Tuberculosis 88, S75-S83. doi: 10.1016/ S1472-9792(08)70038-6

Fallahi-Sichani, M., El-Kebir, M., Marino, S., Kirschner, D. E., and Linderman, J. J. (2011). Multiscale computational modeling reveals a critical role for TNF- $\alpha$ receptor 1 dynamics in tuberculosis granuloma formation. J. Immunol. 186, 3472-3483. doi: 10.4049/jimmunol.1003299

Gillespie, S. H., Crook, A. M., McHugh, T. D., Mendel, C. M., Meredith, S. K., Murray, S. R., et al. (2014). Four-Month Moxifloxacin-Based Regimens for Drug-Sensitive Tuberculosis. N. Engl. J. Med. 371, 1577-1587. doi: 10.1056/ NEJMoa1407426

Global tuberculosis report (2018).

Hartkoorn, R. C., Chandler, B., Owen, A., Ward, S. A., Bertel Squire, S., Back, D. J., et al. (2007). Differential drug susceptibility of intracellular and extracellular tuberculosis, and the impact of P-glycoprotein. Tuberculosis 87, 248-255. doi: 10.1016/j.tube.2006.12.001

Irwin, S. M., Prideaux, B., Lyon, E. R., Zimmerman, M. D., Brooks, E. J., Schrupp, C. A., et al. (2016). Bedaquiline and Pyrazinamide Treatment Responses Are Affected by Pulmonary Lesion Heterogeneity in Mycobacterium tuberculosis Infected C3HeB/FeJ Mice. ACS Infect. Dis. 2, 251-267. doi: 10.1021/ acsinfecdis.5b00127

Jayaram, R., Gaonkar, S., Kaur, P., Suresh, B. L., Mahesh, B. N., Jayashree, R., et al. (2003). Pharmacokinetics-Pharmacodynamics of Rifampin in an Aerosel Infection Model of Tuberculosis. Antimicrob. Agents Chemother. 47, 2118 2124. doi: 10.1128/AAC.47.7.2118

Jayaram, R., Shandil, R. K., Gaonkar, S., Kaur, P., Suresh, B. L., Mahesh, B. N., et al. (2004). Isoniazid pharmacokinetics-pharmacodynamics in an aerosol infection model of tuberculosis. Antimicrob. Agents Chemother. 48, 2951-2957. doi: 10.1128/AAC.48.8.2951-2957.2004

Jindani, A., Doré, C. J., and Mitchison, D. A. (2003). Bactericidal and sterilizing activities of antituberculosis drugs during the first 14 days. Am. J. Respir. Crit. Care Med. 167, 1348-1354. doi: 10.1164/rccm.200210-1125OC

Jindani, A., Harrison, T. S., Nunn, A. J., Phillips, P. P. J., Churchyard, G. J., Charalambous, S., et al. (2014). High-Dose Rifapentine with Moxifloxacin for Pulmonary Tuberculosis. N. Engl. J. Med. 371, 1599-1608. doi: 10.1056/ NEJMoa1314210

Jonsson, S., Davidse, A., Wilkins, J., Van Der Walt, J. S., Simonsson, U. S. H., Karlsson, M. O., et al. (2011). Population pharmacokinetics of ethambutol in South African tuberculosis patients. Antimicrob. Agents Chemother. 55, 42304237. doi: 10.1128/AAC.00274-11

Kinzig-Schippers, M., Tomalik-scharte, D., Jetter, A., Scheidel, B., Jakob, V., Rodamer, M., et al. (2005). Should We Use N-Acetyltransferase Type 2 
Genotyping To Personalize Isoniazid Doses? Antimicrob. Agents Chemother. 49, 1733-1738. doi: 10.1128/AAC.49.5.1733

Kjellsson, M. C., Via, L. E., Goh, A., Weiner, D., Low, K. M., Kern, S., et al. (2012). Pharmacokinetic evaluation of the penetration of antituberculosis agents in rabbit pulmonary lesions. Antimicrob. Agents Chemother. 56, 446-457. doi: 10.1128/AAC.05208-11

Lakshminarayana, S. B., Huat, T. B., Ho, P. C., Manjunatha, U. H., Dartois, V., Dick, T., et al. (2015). Comprehensive physicochemical, pharmacokinetic and activity profiling of anti-TB agents. J. Antimicrob. Chemother. 70, 857-867. doi: $10.1093 / \mathrm{jac} / \mathrm{dku} 457$

Lalande, L., Bourguignon, L., Bihari, S., Maire, P., Neely, M., Jelliffe, R., et al. (2015). Population modeling and simulation study of the pharmacokinetics and antituberculosis pharmacodynamics of isoniazid in lungs. Antimicrob. Agents Chemother. 59, 5181-5189. doi: 10.1128/AAC.00462-15

Lanoix, J. P., Betoudji, F., and Nuermberger, E. (2016). Sterilizing activity of pyrazinamide in combination with first-line drugs in a $\mathrm{C} 3 \mathrm{HeB} / \mathrm{FeJ}$ mouse model of tuberculosis. Antimicrob. Agents Chemother. 60, 1091-1096. doi: 10.1128/AAC.02637-15

Linderman, J. J., Cilfone, N. A., Pienaar, E., Gong, C., and Kirschner, D. E. (2015). A multi-scale approach to designing therapeutics for tuberculosis. Integr. Biol. 7, 591-609. doi: 10.1039/C4IB00295D

Ma, S., Jaipalli, S., Larkins-Ford, J., Lohmiller, J., Aldridge, B. B., Sherman, D. R., et al. (2019). Transcriptomic signatures predict regulators of drug synergy and clinical regimen efficacy against tuberculosis. MBio 10, 1-16. doi: 10.1128/ mBio.02627-19

Magombedze, G., Pasipanodya, J. G., Srivastava, S., Deshpande, D., Visser, M. E., Chigutsa, E., et al. (2018). Transformation Morphisms and Time-to-Extinction Analysis That Map Therapy Duration from Preclinical Models to Patients with Tuberculosis: Translating from Apples to Oranges. Clin. Infect. Dis. 67, S349S358. doi: 10.1093/cid/ciy623

Marino, S., Hogue, I. B., Ray, C. J., and Kirschner, D. E. (2008). A methodology for performing global uncertainty and sensitivity analysis in systems biology. J. Theor. Biol. 254, 178-196. doi: 10.1016/j.jtbi.2008.04.011

McLaren, Z. M., Milliken, A. A., Meyer, A. J., and Sharp, A. R. (2016). Does directly observed therapy improve tuberculosis treatment? More evidence is needed to guide tuberculosis policy. BMC Infect. Dis. 16. doi: 10.1186/s12879016-1862-y

Munro, S. A., Lewin, S. A., Smith, H. J., Engel, M. E., Fretheim, A., and Volmink, J. (2007). Patient Adherence to Tuberculosis Treatment: A Systematic Review of Qualitative Research. PloS Med. 4, 1230-1245. doi: 10.1371/journal.pmed.0040238

Nahid, P., Dorman, S. E., Alipanah, N., Barry, P. M., Brozek, J. L., Cattamanchi, A., et al. (2016). Official American Thoracic Society/Centers for Disease Control and Prevention/Infectious Diseases Society of America Clinical Practice Guidelines: Treatment of Drug-Susceptible Tuberculosis. Clin. Infect. Dis. 63, 853-867. doi: 10.1093/cid/ciw566

Nathan, C., and Barry, C. E.III (2015). TB drug development: Immunology at the table. Immunol. Rev. 264, 308-318. doi: 10.1111/imr.12275

Peloquin, C. A., Velásquez, G. E., Lecca, L., Calderón, R. I., Coit, J., Milstein, M., et al. (2017). Pharmacokinetic Evidence from the HIRIF Trial To Support Increased Doses of Rifampin for Tuberculosis. Antimicrob. Agents Chemother. 61, 1-6. doi: 10.1128/AAC.00038-17

Pienaar, E., Cilfone, N. A., Lin, P. L., Dartois, V., Mattila, J. T., Butler, J. R., et al. (2015a). A computational tool integrating host immunity with antibiotic dynamics to study tuberculosis treatment. J. Theor. Biol. 367, 166-179. doi: 10.1016/j.jtbi.2014.11.021

Pienaar, E., Dartois, V., Linderman, J. J., and Kirschner, D. E. (2015b). In silico evaluation and exploration of antibiotic tuberculosis treatment regimens. $B M C$ Syst. Biol. 9, 1-12. doi: 10.1186/s12918-015-0221-8

Pienaar, E., Matern, W. M., Linderman, J. J., Bader, J. S., and Kirschner, D. E. (2016). Multiscale Model of Mycobacterium tuberculosis Infection Maps Metabolite and Gene Perturbations to Granuloma Sterilization. Infect. Immun. 84, 1650-1669. doi: 10.1128/IAI.01438-15.Editor

Pienaar, E., Sarathy, J., Prideaux, B., Dietzold, J., Dartois, V., Kirschner, D. E., et al. (2017). Comparing efficacies of moxifloxacin, levofloxacin and gatifloxacin in tuberculosis granulomas using a multi-scale systems pharmacology approach. PloS Comput. Biol. 13, e1005650. doi: 10.1371/journal.pcbi.1005650
Pienaar, E., Linderman, J. J., and Kirschner, D. E. (2018). Emergence and selection of isoniazid and rifampin resistance in tuberculosis granulomas. PloS One 13, 1-29. doi: 10.1371/journal.pone.0196322

Prideaux, B., Via, L. E., Zimmerman, M. D., Eum, S., Sarathy, J., O'Brien, P., et al. (2015). The association between sterilizing activity and drug distribution into tuberculosis lesions. Nat. Med. 21, 1223-1227. doi: 10.1038/nm.3937

Pruijn, F. B., Patel, K., Hay, M. P., Wilson, W. R., and Hicks, K. O. (2008). Prediction of tumour tissue diffusion coefficients of hypoxia-activated prodrugs from physicochemical parameters. Aust. J. Chem. 61, 687-693. doi: 10.1071/ $\mathrm{CH} 08240$

Ramakrishnan, L. (2012). Revisiting the role of the granuloma in tuberculosis. Nat. Rev. Immunol. 12, 352-366. doi: 10.1038/nri3211

Sarathy, J. P., Zuccotto, F., Hsinpin, H., Sandberg, L., Via, L. E., Marriner, G. A., et al. (2016). Prediction of Drug Penetration in Tuberculosis Lesions. ACS Infect. Dis. 2, 552-563. doi: 10.1021/acsinfecdis.6b00051

Sarathy, J. P., Via, L. E., Weiner, D., Blanc, L., Boshoff, H., Eugenin, E. A., et al. (2018). Extreme drug tolerance of mycobacterium tuberculosis in Caseum. Antimicrob. Agents Chemother. 62, 1-11. doi: 10.1128/AAC.02266-17

Segovia-Juarez, J. L., Ganguli, S., and Kirschner, D. (2004). Identifying control mechanisms of granuloma formation during $\mathrm{M}$. tuberculosis infection using an agent-based model. J. Theor. Biol. 231, 357-376. doi: 10.1016/j.jtbi.2004.06.031

Sirgel, F. A., Fourie, P. B., Donald, P. R., Padayatchi, N., Rustomjee, R., Levin, J., et al. (2005). The early bactericidal activities of rifampin and rifapentine in pulmonary tuberculosis. Am. J. Respir. Crit. Care Med. 172, 128-135. doi: $10.1164 / \mathrm{rccm} .200411-1557 \mathrm{OC}$

Srivastava, S., and Gumbo, T. (2011). In Vitro and In Vivo Modeling of Tuberculosis Drugs and its Impact on Optimization of Doses and Regimens. Curr. Pharm. Des. 17, 2881-2888. doi: 10.2174/138161211797470192

Steffen, R., Menzies, D., Oxlade, O., Pinto, M., de Castro, A. Z., Monteiro, P., et al. (2010). Patients' costs and cost-effectiveness of tuberculosis treatment in DOT and non-DOT facilities in Rio de Janeiro, Brazil. PloS One 5, 1-7. doi: 10.1371/ journal.pone.0014014

Stott, K. E., Pertinez, H., Sturkenboom, M. G. G., Boeree, M. J., Aarnoutse, R. Ramachandran, G., et al. (2018). Pharmacokinetics of rifampicin in adult TB patients and healthy volunteers: a systematic review and meta-analysis. $J$. Antimicrob. Chemother. 73, 2305-2313. doi: 10.1093/jac/dky152

Strydom, N., Gupta, S. V., Fox, W. S., Via, L. E., Bang, H., Lee, M., et al. (2019). Tuberculosis drugs' distribution and emergence of resistance in patient's lung lesions : A mechanistic model and tool for regimen and dose optimization. PloS Med. 16, e1002773. doi: 10.1371/journal.pmed.1002773

Swaminathan, S., Pasipanodya, J. G., Ramachandran, G., Kumar, A. K. H., Srivastava, S., Deshpande, D., et al. (2016). Drug Concentration Thresholds Predictive of Therapy Failure and Death in Children with Tuberculosis: Bread Crumb Trails in Random Forests. Clin. Infect. Dis. 63, S63-S74. doi: 10.1093/ $\mathrm{cid} / \mathrm{ciw} 471$

Yee, D., Valiquette, C., Pelletier, M., Parisien, I., Rocher, I., and Menzies, D. (2003). Incidence of Serious Side Effects from First-Line Antituberculosis Drugs among Patients Treated for Active Tuberculosis. Am. J. Respir. Crit. Care Med. 167, 1472-1477. doi: 10.1164/rccm.200206-626OC

Zhu, M., Burman, W. J., Starke, J. R., Stambaugh, J. J., Steiner, P., Bulpitt, A. E., et al. (2004). Pharmacokinetics of ethambutol in children and adults with tuberculosis. Int. J. Tuberc. Lung Dis. 8, 1360-1367.

Zimmerman, M., Lestner, J., Prideaux, B., O'Brien, P., Dias-freedman, I., Chen, C., et al. (2017). Ethambutol Partitioning in Tuberculous Pulmonary Lesions Explains Its Clinical Efficacy. Antimicrob. Agents Chemother. 61, 1-12. doi: 10.1128/AAC.00924-17

Conflict of Interest: The authors declare that the research was conducted in the absence of any commercial or financial relationships that could be construed as a potential conflict of interest.

Copyright $\odot 2020$ Cicchese, Dartois, Kirschner and Linderman. This is an open-access article distributed under the terms of the Creative Commons Attribution License (CC $B Y)$. The use, distribution or reproduction in other forums is permitted, provided the original author(s) and the copyright owner(s) are credited and that the original publication in this journal is cited, in accordance with accepted academic practice. No use, distribution or reproduction is permitted which does not comply with these terms. 